\title{
The Arc-Sine Laws for the Skew Brownian Motion and Their Interpretation
}

\author{
Ivan H. Krykun ${ }^{1,2}$ \\ ${ }^{1}$ Pervomajs'k Branch of Admiral Makarov National Shipbuilding University, Pervomajs'k, Ukraine \\ ${ }^{2}$ Institute of Applied Mathematics and Mechanics of NAS of Ukraine, Slovjans'k, Ukraine \\ Email: iwanko@i.ua
}

How to cite this paper: Krykun, I.H (2018) The Arc-Sine Laws for the Skew Brownian Motion and Their Interpretation. Journal of Applied Mathematics and Physics, 6, 347-357.

https://doi.org/10.4236/jamp.2018.62033

Received: January 5, 2018

Accepted: February 5, 2018

Published: February 8, 2018

Copyright (C) 2018 by author and Scientific Research Publishing Inc. This work is licensed under the Creative Commons Attribution International License (CC BY 4.0).

http://creativecommons.org/licenses/by/4.0/

\begin{abstract}
We consider the skew Brownian motion as a solution of some stochastic differential equation. We prove for the skew Brownian motion the analogues of the arc-sine laws for Wiener process. Unlike of existing results, we are forced to consider a stochastic differential equation with discontinuous diffusion coefficient. Possible interpretations of obtained results are suggested.
\end{abstract}

\section{Keywords}

Skew Brownian Motion, Local Time, Arc-Sine Law

\section{Introduction}

Let $I_{\{A\}}(x)$ be the indicator function of set $A$. Levy in [1] proved such result: Theorem 1. Let $\{w(t), t \in[0,1]\}$ be a standard Wiener process. Then for $0 \leq x \leq 1$

$$
\mathrm{P}\left\{\int_{0}^{1} I_{\{(0, \infty)\}}(w(t)) \mathrm{d} t \leq x\right\}=\frac{2}{\pi} \arcsin \sqrt{x} .
$$

Later one received other similar results where appear arc-sine function. They were called the arc-sine law, particularly result of the theorem 1 was named "the first arc-sine law". One consequence of the work [1] is the following result:

Theorem 2. ([2], theorem 3.27). Let

$$
L \stackrel{\text { def }}{=} \sup \{t \leq 1: w(t)=0\}
$$

be the last zero of Wiener process until the instant $t=1$. Then (for $0 \leq x \leq 1$ )

$$
\mathrm{P}\{L \leq x\}=\frac{2}{\pi} \arcsin \sqrt{x}
$$

Theorem 3. [3]. Let 


$$
Z \stackrel{\text { def }}{=} \arg \max _{t \in[0,1]}\{w(t)\}
$$

be the instant of hitting Wiener process its maximum for time $t \in[0,1]$. Then (for $0 \leq x \leq 1$ )

$$
\mathrm{P}\{Z \leq x\}=\frac{2}{\pi} \arcsin \sqrt{x}
$$

Theorem 4. ([2], theorem 3.27). Let

$$
R \stackrel{\text { def }}{=} \sup \{t \geq 1: w(t)=0\}
$$

be the first zero of Wiener process after the instant $t=1$. Then (for $x \geq 1$ )

$$
\mathrm{P}\{R \geq x\}=\frac{2}{\pi} \arcsin \sqrt{\frac{1}{x}} .
$$

Among other researches that contain results of arc-sine law type for different functionals we should mention paper [4] where the result such as Theorem 1 for a process obtained by pasting together two Brownian motion processes was obtained.

In the article [5], besides another results, the analogue of Theorem 1 for the Brownian motion with linear drift was proved.

In ([6], remark 1) the result similar to Theorem 1 was proved for solution of stochastic equation

$$
\eta(t)=x+\int_{0}^{t} \sigma(\eta(s)) \mathrm{d} w(s),
$$

if there exist such finite limits

$$
\lim _{x \rightarrow \pm \infty} \frac{1}{x} \int_{0}^{x} \frac{\mathrm{d} y}{\sigma^{2}(y)} .
$$

Work [7] is devoted to generalization of results of Theorems 1 and 2 to symmetric Bessel process with index $v \in(-1,0)$ starting from 0 .

The article [8] contains results analogous to Theorems 1 and 2 for process $\xi_{u}(t)$-Brownian bridge of length $u$. In [9] author investigated joint distribution of functionals from Theorems 1, 2, 3 .

In the paper [10] is considered asymptotic behavior of probability

$$
\mathrm{P}\left\{\int_{0}^{1} I_{\{w(t)>0\}} \mathrm{d} t \leq x\right\}
$$

if $x \rightarrow 0$.

The work [11] contains results such as Theorem 3 for the instant of hitting by solution of homogeneous stochastic equation

$$
X(t)=X_{0}+\int_{0}^{t} b(X(s)) \mathrm{d} s+\int_{0}^{t} \sigma(X(s)) \mathrm{d} w(s)
$$

its maximum for time $[0, T]$ and for $\theta$-the first instant when for functional $S_{t}=\frac{1}{t} \int_{0}^{t} w(s) \mathrm{d} s$ equality $S_{T}=w(\theta)$ fulfils.

The article [12] is devoted to proof of results such as Theorem 1 for the telegraph process. 
Papers [7] and [13] contain summary of further results which generalized results of Levy and others.

In this paper we consider a skew Brownian motion. It was defined by Ito \& McKean [14] and later was investigated by many other authors. We mention works such scientists as Harrison \& Shepp [15] and Le Gall [16] where skew Brownian motion was connected with solution of stochastic process involving the local time. Moreover, in papers [16] [17] [18] were obtained formulae which connect solutions of stochastic equations involving the local time with solutions of Ito's stochastic equations. Two recent entry points into the literature of occupation times and related functionals of skew Brownian motion are [19] and [20].

In this paper for the skew Brownian motion arc-sine laws analogous Theorems $1-4$ for Brownian motion are proved. We consider Ito's stochastic equation with discontinuous diffusion coefficient unlike existing results.

This paper is organized as follows: the second section contains denotation and definition of considered functionals and main results-Theorems $5-8$. In the third section Theorems $5-8$ and auxiliary Lemma are proved. The fourth section contains some interpretation of obtained results.

\section{Main Results}

Let us consider the skew Brownian motion as a solution of stochastic equation involving the local time

$$
\xi(t)=\beta L^{\xi}(t, 0)+w(t), t \geq 0 .
$$

If $|\beta| \leq 1$ then there are the unique strong solution of Equation (1) [15], i.e. on the probability space $\left(\Omega, \mathfrak{F}, \mathfrak{F}_{t}, \mathrm{P}\right)$ with flow of $\sigma$-algebras $\mathfrak{F}_{t}, t \geq 0$ and given standard one-dimensional Wiener process $\left(w(t), \mathfrak{F}_{t}\right)$, there exist a continuous semi-martingale $\left(\xi(t), \mathfrak{F}_{t}\right)$ such as a symmetric local time

$$
L^{\xi}(t, 0)=\lim _{\delta \rightarrow 0} \frac{1}{2 \delta} \int_{0}^{t} I_{(-\delta, \delta)}(\xi(s)) \mathrm{d} s
$$

exists almost surely and Equation (1) fulfils almost surely.

We denote

$$
\begin{gathered}
\Phi(x)=\frac{1}{\sqrt{2 \pi}} \int_{-\infty}^{x} \exp \left\{-\frac{y^{2}}{2}\right\} \mathrm{d} y \\
\mathrm{P}^{x}\{\xi(t) \in A\}=\mathrm{P}\{\xi(t) \in A \mid \xi(0)=x\}=\int_{A} p_{t}(x, y) \mathrm{d} y, \text { for set } A \in \mathfrak{F} .
\end{gathered}
$$

The main results of this article are four following theorems.

Theorem 5. Let $\xi$ be the skew Brownian motion, defined by (1) and constant $|\beta|<1$. Then

$$
\mathrm{P}\left\{\frac{1}{T} \int_{0}^{T} I_{\{\xi(t)>0\}} \mathrm{d} t \leq x\right\}=\left\{\begin{array}{lc}
0, & \text { if } x \leq 0, \\
\frac{2}{\pi} \arcsin \sqrt{\frac{(1-\beta)^{2} x}{(1+\beta)^{2}-4 \beta x}}, & \text { if } x \in(0,1), \\
1, & \text { if } x \geq 1 .
\end{array}\right.
$$


Remark 1. It's clear if $\beta=0$ then from Theorem 5 we have result for Wiener process, i.e. Theorem 1.

Remark 2. It's well known [15] that if $\beta=1$ then distributions of skew Brownian motion coincide with distributions of process $|w(t)|$ and if $\beta=-1-$ with distributions of process $-|w(t)|$. So we should expect that for $\beta \rightarrow 1$ the probability $\mathrm{P}\left\{\frac{1}{T} \int_{0}^{T} I_{\{\xi(t)>0\}} \mathrm{d} t \leq x\right\}$ will tend to 0 for $0<x<1$, and for $\beta \rightarrow-1$ the probability $\mathrm{P}\left\{\frac{1}{T} \int_{0}^{T} I_{\{\xi(t)>0\}} \mathrm{d} t \leq x\right\}$ will tend to 1 for $0<x<1$. Obviously that the same result follows from formula (2).

Let us define

$$
\begin{gathered}
N_{T} \stackrel{\text { def }}{=} \sup \{t \in[0, T]: \xi(t)=0\} ; \\
M_{T} \stackrel{\text { def }}{=} \sup _{t \in[0, T]}\{\xi(t)\}, \quad m_{T} \stackrel{\text { def }}{=} \inf _{t \in[0, T]}\{\xi(t)\} ; \\
\tau_{T, x}=\inf _{t \in[0, T]}\{\xi(t)=x\}, \quad \text { here set inf }\{\varnothing\}=T+1 .
\end{gathered}
$$

It's clear that for $C>0$

$$
\left\{\omega: M_{T} \geq C\right\}=\left\{\omega: \tau_{T, C} \leq T\right\},
$$

and for $C<0$

$$
\left\{\omega: m_{T} \leq C\right\}=\left\{\omega: \tau_{T, C} \leq T\right\} .
$$

Theorem 6. Let $\xi$ be the skew Brownian motion, defined by (1) and constant $|\beta|<1$. Then for $0 \leq x \leq T$

$$
\mathrm{P}\left\{N_{T} \leq x\right\}=\frac{2}{\pi} \arcsin \sqrt{\frac{x}{T}} .
$$

Let us denote

$$
\begin{gathered}
Z_{T} \stackrel{\text { def }}{=} \arg \max _{0 \leq t \leq T} \xi(t), \\
R_{T} \stackrel{\text { def }}{=} \inf _{t>T}\{\xi(t)=0\} .
\end{gathered}
$$

Theorem 7. Let $\xi$ be the skew Brownian motion, defined by (1) and constant $|\beta|<1$. Then for $0 \leq x \leq T$

$$
\mathrm{P}\left\{Z_{T} \leq x\right\}=\frac{2}{\pi} \arcsin \sqrt{\frac{x}{T}} .
$$

Theorem 8. Let $\xi$ be the skew Brownian motion, defined by (1) and constant $|\beta|<1$. Then for $x \geq 0$

$$
\mathrm{P}\left\{R_{T}>T+x\right\}=\frac{2}{\pi} \arcsin \sqrt{\frac{T}{T+x}} .
$$

\section{Proofs of Theorems 5 - 8}

\section{We denote}




$$
\operatorname{sgn} x= \begin{cases}-1, & \text { if } x<0 \\ 0, & \text { if } x=0 \\ 1, & \text { if } x>0\end{cases}
$$

Solution of Equation (1) is closely connected with solution of some Ito's stochastic equation. Namely let's define the function

$$
\kappa(x)= \begin{cases}(1-\beta) x, & x<0, \\ (1+\beta) x, & x \geq 0,\end{cases}
$$

and so

$$
\varphi(x)= \begin{cases}\frac{x}{1-\beta}, & x<0 \\ \frac{x}{1+\beta}, & x \geq 0\end{cases}
$$

be inverse function to $\kappa(x)$.

Now we consider such Ito's stochastic equation

$$
\eta(t)=\int_{0}^{t} \frac{\mathrm{d} w(s)}{1+\beta \operatorname{sgn} \eta(s)}, t \geq 0 .
$$

The diffusion coefficient of this process is a discontinuous function of bounded variation so from ([21], Theorem) it follows the existence of strong solution of Equation (7).

It is well known [17] that $\eta(t)=\varphi(\xi(t))$ or $\xi(t)=\kappa(\eta(t))$. Remark that $\xi(t)$ and $\eta(t)$ have the same sign.

Proof of Theorem 5. For some constant $|\beta|<1$ for the processes $\xi$ and $\eta$ we have such property of their occupation times:

$$
\frac{1}{T} \int_{0}^{T} I_{\{\xi(t)>0\}} \mathrm{d} t=\frac{1}{T} \int_{0}^{T} I_{\{\kappa(\eta(t))>0\}} \mathrm{d} t=\frac{1}{T} \int_{0}^{T} I_{\{\eta(t)>0\}} \mathrm{d} t .
$$

We denote function $B(x)$ :

$$
B(x)= \begin{cases}\frac{1}{1-\beta}, & \text { if } x<0, \\ \frac{1}{1+\beta}, & \text { if } x \geq 0,\end{cases}
$$

and consider equation

$$
x(t)=\int_{0}^{t} B(x(s)) \mathrm{d} w(s), t \geq 0 .
$$

From [21] it follows the existence of unique strong solution of Equation (9). Because processes $\eta(t)$ and $x(t)$ are different only at point 0 and both processes $\eta(t)$ and $x(t)$ spend zero time at point 0 so we have equality

$$
\int_{0}^{T} I_{\{\eta(t)>0\}} \mathrm{d} t=\int_{0}^{T} I_{\{\eta(t) \geq 0\}} \mathrm{d} t=\int_{0}^{T} I_{\{x(t) \geq 0\}} \mathrm{d} t .
$$

For the functional $\frac{1}{T} \int_{0}^{T} I_{\{x(t) \geq 0\}} \mathrm{d} t$ we may use ([22], theorem 13, p. 149). From it and formulae (8)-(10) we get the statement of Theorem 5. 
Remark 3. It follows from Theorem 1 that for Wiener process $w(t)$

$$
\mathrm{P}\left\{\frac{1}{T} \int_{0}^{T} I_{\{w(t)>0\}} \mathrm{d} t \leq \frac{1}{2}\right\}=\frac{1}{2} .
$$

We can compare this result with analogous one for skew Brownian motion. It follows from Theorem 5 that

$$
\begin{gathered}
\mathrm{P}\left\{\frac{1}{T} \int_{0}^{T} I_{\{\xi(t)>0\}} \mathrm{d} t \leq \frac{1}{2}\right\}=\frac{2}{\pi} \arcsin \sqrt{\frac{(1-\beta)^{2}}{2+2 \beta^{2}}}, \\
\mathrm{P}\left\{\frac{1}{T} \int_{0}^{T} I_{\{\xi(t)>0\}} \mathrm{d} t \leq \frac{(1+\beta)^{2}}{2+2 \beta^{2}}\right\}=\frac{1}{2} .
\end{gathered}
$$

Lemma. For $x>0$ following equalities take place

$$
\begin{aligned}
\mathrm{P}\left\{M_{T} \geq x\right\} & =\frac{2}{1+\beta} \mathrm{P}\{\xi(T) \geq x\}=2\left(1-\Phi\left(\frac{x}{\sqrt{T}}\right)\right) \\
& =\int_{0}^{T} \frac{1}{\sqrt{2 \pi z^{3}}} \exp \left\{-\frac{x^{2}}{2 z}\right\} x \mathrm{~d} z,
\end{aligned}
$$

and for $x<0$

$$
\begin{aligned}
\mathrm{P}\left\{m_{T} \leq x\right\} & =\frac{2}{1-\beta} \mathrm{P}\{\xi(T) \leq x\}=2\left(1-\Phi\left(\frac{|x|}{\sqrt{T}}\right)\right) \\
& =\int_{0}^{T} \frac{1}{\sqrt{2 \pi z^{3}}} \exp \left\{-\frac{x^{2}}{2 z}\right\}|x| \mathrm{d} z .
\end{aligned}
$$

Proof. We firstly consider the case $x>0$. By the multiplication rule of probability, definition of $M_{T}$ and property (3):

$$
\begin{aligned}
\mathrm{P}\{\xi(T) \geq x\} & =\mathrm{P}\left\{\xi(T) \geq x \mid M_{T} \geq x\right\} \mathrm{P}\left\{M_{T} \geq x\right\} \\
& =\mathrm{P}\left\{\xi(T) \geq x \mid \tau_{T, x} \leq T\right\} \mathrm{P}\left\{M_{T} \geq x\right\} .
\end{aligned}
$$

Now consider the first multiplicand in the right hand side of equality (13). From ([22], theorem 5, p. 168) it follows homogeneousness and strong Markov property of skew Brownian motion. Then by ([23], remark 2, p. 108) for process $\tilde{\xi}(t)=\xi\left(t+\tau_{T, x}\right)-\xi\left(\tau_{T, x}\right)$, such as its distributions coincided with distributions of process $\xi(t)$ and $\tilde{\xi}(0)=0$ we have:

$$
\begin{aligned}
& \mathrm{P}\left\{\xi(T) \geq x \mid \tau_{T, x} \leq T\right\} \\
& =\mathrm{P}\left\{\xi(T)-x \geq 0 \mid \tau_{T, x} \leq T\right\}=\mathrm{P}\left\{\xi(T)-\xi\left(\tau_{T, x}\right) \geq 0 \mid \tau_{T, x} \leq T\right\} \\
& =\mathrm{P}\left\{\tilde{\xi}\left(T-\tau_{T, x}\right) \geq 0 \mid \tau_{T, x} \leq T\right\}=\mathrm{P}\left\{\tilde{\xi}\left(t^{*}\right) \geq 0 \mid t^{*} \geq 0\right\}=\frac{1+\beta}{2} .
\end{aligned}
$$

The last equality follows from properties of skew Brownian motion ([22], p. 169). So from (13) it follows the first equality in (11).

Further we use the formula for transition probability density function of skew Brownian motion (22, theorem 6, p. 168). Then for $x>0$ 


$$
\begin{aligned}
\mathrm{P}\left\{M_{T} \geq x\right\} & =\frac{2}{1+\beta} \mathrm{P}\{\xi(T) \geq x\} \\
& =\frac{2}{1+\beta} \int_{x}^{\infty} \frac{1+\beta}{\sqrt{2 \pi T}} \exp \left\{-\frac{y^{2}}{2 T}\right\} \mathrm{d} y \\
& =2\left(1-\Phi\left(\frac{x}{\sqrt{T}}\right)\right)=2 \int_{x}^{\infty} \frac{1}{\sqrt{2 \pi T}} \exp \left\{-\frac{y^{2}}{2 T}\right\} \mathrm{d} y \\
& =\int_{0}^{T} \frac{1}{\sqrt{2 \pi z^{3}}} \exp \left\{-\frac{x^{2}}{2 z}\right\} x \mathrm{~d} z .
\end{aligned}
$$

The result for $\mathrm{P}\left\{m_{T} \leq x\right\}$ can be proved analogously.

Corollary. Now we compare results of Lemma with known results about distributions of maximum and minimum of Wiener process ([2], theorems 3.15, 3.17) and conclude that distribution of maximum (minimum) of skew Brownian motion not depend from skewness coefficient $\beta$ and coincide with distribution of maximum (respectively minimum) of Wiener process.

Proof of Theorem 6. By the Markov property of process $\xi$, ChapmanKolmogorov equation, properties (3), (4) and results of the Lemma we have:

$$
\begin{aligned}
\mathrm{P}\left\{N_{T} \leq x\right\} & =\int_{-\infty}^{+\infty} p_{x}(0, y) \mathrm{P}^{y}\left(\tau_{T, 0}>T-x\right) \mathrm{d} y \\
& =\int_{-\infty}^{+\infty} p_{x}(0, y) \mathrm{P}^{0}\left(\tau_{T, y}>T-x\right) \mathrm{d} y \\
& =\int_{-\infty}^{0} p_{x}(0, y) \mathrm{P}^{0}\left(m_{T-x}>y\right) \mathrm{d} y+\int_{0}^{+\infty} p_{x}(0, y) \mathrm{P}^{0}\left(M_{T-x}<y\right) \mathrm{d} y \\
& =\int_{-\infty}^{0} \frac{1-\beta}{\sqrt{2 \pi x}} \exp \left\{-\frac{y^{2}}{2 x}\right\} \mathrm{P}\left(m_{T-x}>y\right) \mathrm{d} y \\
& +\int_{0}^{+\infty} \frac{1+\beta}{\sqrt{2 \pi x}} \exp \left\{-\frac{y^{2}}{2 x}\right\} \mathrm{P}\left(M_{T-x}<y\right) \mathrm{d} y=I_{1}+I_{2} . \\
I_{1}= & (1-\beta) \int_{-\infty}^{0} \frac{1}{\sqrt{2 \pi x}} \exp \left\{-\frac{y^{2}}{2 x}\right\} P^{0}\left(m_{T-x}>y\right) \mathrm{d} y \\
= & (1-\beta) \int_{-\infty}^{0} \frac{1}{\sqrt{2 \pi x}} \exp \left\{-\frac{y^{2}}{2 x}\right\} \int_{T-x}^{+\infty} \frac{1}{\sqrt{2 \pi z^{3}}} \exp \left\{-\frac{y^{2}}{2 z}\right\}|y| \mathrm{d} z \mathrm{~d} y \\
= & -\frac{1-\beta}{2 \pi} \int_{T-x}^{+\infty} \frac{1}{\sqrt{x z^{3}}} \int_{-\infty}^{0} y \exp \left\{-\frac{y^{2}(x+z)}{2 x z}\right\} \mathrm{d} y \mathrm{~d} z \\
= & -\frac{1-\beta}{2 \pi} \int_{T-x}^{+\infty} \frac{1}{\sqrt{x z^{3}}}\left(-\left.\frac{x z}{x+z} \exp \left\{-\frac{y^{2}(x+z)}{2 x z}\right\}\right|_{y=-\infty} ^{0}\right) \mathrm{d} z \\
= & \frac{\beta-1}{2 \pi} \int_{T-x}^{+\infty} \frac{1}{\sqrt{x z^{3}}}\left(-\frac{x z}{x+z}\right) \mathrm{d} z=\frac{\beta-1}{2 \pi} \int_{\frac{x}{T}}^{0} \frac{1}{\sqrt{t(1-t)}} \mathrm{d} t \\
= & \frac{1-\beta}{\pi} \arcsin \sqrt{\frac{x}{T}} .
\end{aligned}
$$

Analogously

$$
I_{2}=(1+\beta) \int_{0}^{+\infty} \frac{1}{\sqrt{2 \pi x}} \exp \left\{-\frac{y^{2}}{2 x}\right\} \mathrm{P}^{0}\left(M_{T-x}<y\right) \mathrm{d} y=\frac{1+\beta}{\pi} \arcsin \sqrt{\frac{x}{T}} .
$$


So we get (5).

Proof of Theorem 7.

$$
\begin{aligned}
\mathrm{P}\left\{Z_{T} \leq x\right\} & =\mathrm{P}\left\{\max _{[0, x]} \xi(u)>\max _{[x, T]} \xi(u)\right\} \\
& =\mathrm{P}\left\{\max _{[0, x]}(\xi(u)-\xi(x))>\max _{[x, T]}(\xi(u)-\xi(x))\right\} .
\end{aligned}
$$

Analogously to proof of the Lemma for processes $\xi_{1}(t)=\xi(x-t)-\xi(x)$ and $\xi_{2}(t)=\xi(x+t)-\xi(x)$ with $\xi_{1}(0)=\xi_{2}(0)=0$ distribution of maximum (and minimum) each of them coincide by Corollary with distribution of maximum (and minimum) of Wiener process $w(t)$ with $w(0)=0$ and we have

$$
\begin{aligned}
\mathrm{P}\left\{Z_{T} \leq x\right\} & =\mathrm{P}\left\{\max _{[0, x]} \xi_{1}(u)>\max _{[0, T-x]} \xi_{2}(u)\right\} \\
& =\mathrm{P}\left\{\max _{[0, x]} w(u)>\max _{[0, T-x]} w(u)\right\} .
\end{aligned}
$$

So statement of Theorem 7 can be proved in the same way as analogously proof of such result for Wiener process (for example see [24], theorem 5.26).

Proof of Theorem 8. By the Markov property of process $\xi$, ChapmanKolmogorov equation, properties (3), (4) and results of the Lemma we have:

$$
\begin{aligned}
& \mathrm{P}\left\{R_{T}>T+x\right\}=\int_{-\infty}^{+\infty} p_{T}(0, y) \mathrm{P}^{y}\left(\tau_{T, 0}>x\right) \mathrm{d} y=\int_{-\infty}^{+\infty} p_{T}(0, y) \mathrm{P}^{0}\left(\tau_{T,-y}>x\right) \mathrm{d} y \\
& =\int_{-\infty}^{0} p_{T}(0, y) \mathrm{P}^{0}\left(M_{x}<-y\right) \mathrm{d} y+\int_{0}^{+\infty} p_{T}(0, y) \mathrm{P}^{0}\left(m_{x}>-y\right) \mathrm{d} y \\
& =\int_{-\infty}^{0} \frac{1-\beta}{\sqrt{2 \pi T}} \exp \left\{-\frac{y^{2}}{2 T}\right\} \mathrm{P}\left(M_{x}<-y\right) \mathrm{d} y \\
& +\int_{0}^{+\infty} \frac{1+\beta}{\sqrt{2 \pi T}} \exp \left\{-\frac{y^{2}}{2 T}\right\} \mathrm{P}\left(m_{x}>-y\right) \mathrm{d} y \\
& =I_{3}+I_{4} \text {. } \\
& I_{3}=(1-\beta) \int_{-\infty}^{0} \frac{1}{\sqrt{2 \pi T}} \exp \left\{-\frac{y^{2}}{2 T}\right\} P^{0}\left(M_{x}<-y\right) \mathrm{d} y \\
& =(1-\beta) \int_{-\infty}^{0} \frac{1}{\sqrt{2 \pi T}} \exp \left\{-\frac{y^{2}}{2 T}\right\} \int_{x}^{+\infty} \frac{1}{\sqrt{2 \pi z^{3}}} \exp \left\{-\frac{y^{2}}{2 z}\right\}(-y) \mathrm{d} z \mathrm{~d} y \\
& =-\frac{1-\beta}{2 \pi} \int_{x}^{+\infty} \frac{1}{\sqrt{T z^{3}}} \int_{-\infty}^{0} y \exp \left\{-\frac{y^{2}(T+z)}{2 T z}\right\} \mathrm{d} y \mathrm{~d} z \\
& =-\frac{1-\beta}{2 \pi} \int_{x}^{+\infty} \frac{1}{\sqrt{T z^{3}}}\left(-\left.\frac{T z}{T+z} \exp \left\{-\frac{y^{2}(T+z)}{2 T z}\right\}\right|_{y=-\infty} ^{0}\right) \mathrm{d} z \\
& =\frac{\beta-1}{2 \pi} \int_{x}^{+\infty} \frac{1}{\sqrt{T z^{3}}}\left(-\frac{T z}{T+z}\right) \mathrm{d} z=\frac{\beta-1}{2 \pi} \int_{\frac{T}{T+x}}^{0} \frac{1}{\sqrt{y(1-y)}} \mathrm{d} y \\
& =\frac{1-\beta}{\pi} \arcsin \sqrt{\frac{T}{T+x}} \text {. }
\end{aligned}
$$

Analogously

$$
I_{4}=\frac{1+\beta}{\pi} \arcsin \sqrt{\frac{T}{T+x}},
$$


and so we get (6).

\section{Some Possible Interpretation of Obtained Results}

Now let's mention numerous researches of price dynamics of some economic asset-rate of currencies, stocks, goods, ForEx market etc.-which contain attempts to predict this price dynamics. If investor consider market as stable and homogeneous and he/she suppose that fluctuations of economic asset rate are caused only by random causes then for prediction of the rate currency or other economic asset investor may try to use an arc-sin law for Wiener process.

Further in studies devoted to investigation of pricing different goods, currencies, securities we often meet an idea of "psychological barrier", i.e. the price, after reaching of it we observe a significant change of attitude to this "goods" - an additional growth or fall the demand on it.

From other side it's well known ([22], p. 139) that local time of one-dimensional random process is like some partially permeable "membrane" such as path of one-dimensional random process with local time after reaching the level of local time with probability $\frac{1+\beta}{2}$ will go up and with probability $\frac{1-\beta}{2}$ it will go down.

That's why we suppose that skew Brownian motion can be considered as a model of behavior of rate currencies, stocks or other economic assets with semipermeable barrier-it may be caused by psychological, normative or other causes. So results of this article can be used for prediction of the rate currency or other economic asset.

Moreover by the same causes it's naturally to suppose that results of this article can be used for modelling and prediction the behavior of other processes, such as demographic, electoral, biological ones.

\section{Acknowledgements}

Sincere thanks to the members of JAMP for their professional performance, and special thanks to managing editor Jasmyn Chen for a rare attitude of high quality.

\section{References}

[1] Lévy, P. (1939) Sur certains processus stochastiques homogenes. Compositio Mathematica, 7, 283-339. (In French)

[2] Klebaner, F.C. (2005) Introduction to Stochastic Calculus with Applications. 2nd Edition, Imperial College Press, London. https://doi.org/10.1142/p386

[3] Lévy, P. (1965) Processus Stochastiques et Mouvement Brownienw. Gauthier-villars, Paris. (In French)

[4] Efimenko, S.V. and Portenko, M.I. (1989) Sojourn Time Type Functionals of a Process Obtained by Pasting Together Two Brownian Motion Processes. Ukrainian Mathematical Journal, 41, 25-29. https://doi.org/10.1007/BF01060642 
[5] Pechtl, A. (1999) Distributions of Occupation Times of Brownian Motion with Drift. Journal of Applied Mathematics, 3, 41-62. https://doi.org/10.1155/S1173912699000036

[6] Khasminskii, R. (1999) Arcsin Law and One Generalization. Acta Applicandae Mathematicue, 58, 151-157. https://doi.org/10.1023/A:1006336605269

[7] Graversen, S.E. and Vuolle-Apiala, J. (2000) On Paul Levy's Arc Sine Law and Shiga-Watanabe's Time Inversion Result. Probability and Mathematical Statlstics, 20, 63-73.

[8] Nikitin, Y. and Orsinger, E. (2000) The Intermediate Arc-Sine Law. Statistics \& Probability Letters, 49, 119-125. https://doi.org/10.1016/S0167-7152(00)00038-9

[9] Mishchenko, A.S. (2003) The Three Arcsine Laws. Russian Mathematical Surveys, 58, 1208-1209. https://doi.org/10.1070/RM2003v058n06ABEH000686

[10] Kasahara, Y. and Yano, Y. (2005) On a Generalized Arc-Sine Law for One-Dimensional Diffusion Processes. Osaka Journal of Mathematics, 42, 1-10.

[11] Abundo, M. (2006) The Arc-Sine Law for the First Instant at Which a Diffusion Process Equals the Ultimate Value of a Functional. International Journal of Pure and Applied Mathematics, 30, 13-22.

[12] Bogachev, L. and Ratanov, N. (2011) Occupation Time Distributions for the Telegraph Process. Stochastic Processes and Their Applications, 121, 1816-1844. https://doi.org/10.1016/j.spa.2011.03.016

[13] Pitman, J. and Yor, M. (1992) Arcsine Laws and Interval Partitions Derived from a Stable Subordinator. Proceedings of the London Mathematical Society, 65, 326-356. https://doi.org/10.1112/plms/s3-65.2.326

[14] Ito, K. and McKean, H. (1963) Brownian Motions on a Half Line. Illinois Journal of Mathematics, 7, 181-231.

[15] Harrison, J.M. and Shepp, L.A. (1981) On Skew Brownian Moution. The Annals of Probability, 9, 309-313. https://doi.org/10.1214/aop/1176994472

[16] Le Gall, J.-F. (1983) One-Dimensional Stochastic Equations Involving the Local Times of the Unknown Process. Proceedings of the International Conference on Stochastic Analysis and Applications, Swansea, 11-15 April 1983, Lecture Notes in Mathematics, Vol. 1095, 51-82. https://doi.org/10.1007/BFb0099122

[17] Makhno, S.Y. (2001) A Limit Theorem for Stochastic Equations with Local Time. Theory of Probability and Mathematical Statistics, 64, 123-127.

[18] Engelbert, H.-J. and Schmidt, W. (1991) Strong Markov Continuous Local Martingales and Solutions of One-Dimensional Stochastic Differential Equations, III. Mathematische Nachrichten, 151, 149-197. https://doi.org/10.1002/mana.19911510111

[19] Appuhamillage, T., Bokil, V., Thomann, E., Waymire, E. and Wood, B. (2011) Occupation and Local Times for Skew Brownian Motion with Applications to Dispersion across an Interface. The Annals of Applied Probability, 21, 183-214. https://doi.org/10.1214/10-AAP691

[20] Etore, P. and Martinez, M. (2013) Exact Simulation of One-Dimensional Stochastic Differential Equations Involving the Local Time at Zero of the Unknown Process. Monte Carlo Methods and Applications, 19, 41-71.

[21] Nakao, S. (1972) On the Pathwise Uniqueness of Solutions of One Dimensional Stochastic Differential Equations. Osaka Journal of Mathematics, 9, 513-518.

[22] Makhno, S.Y. (2012) Stochastic Equations. Limit Theorems. Problems and Methods: Mathematics, Mechanics, Cybernetics. Vol. 6. Naukova dumka, Kyiv. (In 
Russian)

[23] Gikhman, I.I. and Skorokhod, A.V. (1972) Stochastic Differential Equations. Springer-Verlag, New York, Heidelberg. https://doi.org/10.1007/978-3-642-88264-7

[24] Mörters, P. and Peres, Y. (2010) Brownian Motion. Cambridge University Press, Cambridge. https://doi.org/10.1017/CBO9780511750489 